\title{
Circulating Nucleic Acids in Plasma and Serum: An Overview
}

\author{
Y. M. DENNIS LO \\ Department of Chemical Pathology and Institute of Molecular Oncology, The Chinese \\ University of Hong Kong, Prince of Wales Hospital, Shatin, New Territories, Hong Kong \\ Special Administrative Region, China
}

\begin{abstract}
The recent interest in nucleic acids in plasma and serum has opened up numerous new areas of investigation and new possibilities for molecular diagnosis. In oncology, tumor-derived genetic changes, epigenetic alterations, and viral nucleic acids have been found in the plasma/serum of cancer patients. These findings have important implications for the detection, monitoring, and prognostication of many types of malignancies. In prenatal diagnosis, the discovery of fetal DNA in maternal plasma and serum has provided a noninvasive source of fetal genetic material for analysis. This development has important implications for the realization of noninvasive prenatal diagnosis and has provided new methods for the monitoring of pregnancy-associated disorders. Plasma DNA technology has also found recent applications in the fields of organ transplantation, posttrauma monitoring, and infectious agent detection. Future areas of study include circulating RNA in plasma and the elucidation of the biology of release, clearance, and possible functionality of plasma nucleic acids.
\end{abstract}

KeYwords: Plasma DNA; Overview; Cancer detection; Noninvasive prenatal diagnosis; Circulating RNA; Organ transplantation

\section{INTRODUCTION}

The recent development of techniques in molecular biology has given us many powerful tools for molecular analysis. However, with the exception of viral nucleic acid detection in plasma, most of these analyses are performed using nucleic acids extracted from nucleated cells. This review explores the recent developments in the biology and diagnostic applications of nucleic acids that are found outside cells, specifically in the plasma and serum.

Address for correspondence: Y. M. Dennis Lo, Department of Chemical Pathology, Room 38023, 1/F Clinical Sciences Building, Prince of Wales Hospital, 30-32 Ngan Shing Street, Shatin, New Territories, Hong Kong Special Administrative Region, China. Voice: +852 2632 2563; fax: +852 21946171.

loym@cuhk.edu.hk 


\section{LANDMARK DEVELOPMENTS}

The discovery of extracellular nucleic acids in the circulation was reported by Mandel and Métais in 1948. ${ }^{1}$ These investigators observed the presence of circulating DNA and RNA in the plasma of healthy and sick individuals. This work was particularly remarkable as it was only a few years following the demonstration that DNA is the material of inheritance, and this report even preceded the classical paper on the double-helical structure of DNA by Watson and Crick. It was unfortunate, therefore, to observe that there was essentially no interest in circulating nucleic acids in the subsequent decade following this pioneering work.

Research on circulating DNA resumed in the 1960s following the discovery of high levels of circulating DNA in patients with systemic lupus erythematosus (SLE). ${ }^{2}$ Further interest in this field was developed in the 1970 s following the demonstration that increased concentrations of DNA in the serum could be detected in patients with cancer. ${ }^{3}$ It was further shown that the concentration of circulating DNA was higher in individuals with metastatic disease and, in some cases, the levels of circulating DNA decreased with successful anticancer therapy. ${ }^{3}$ Due to technological limitations at that time, the precise cellular origin of the extracellular DNA in cancer patients could not be determined.

In 1989, Stroun et al. suggested that the circulating DNA that is found in cancer patients demonstrates certain characteristics of tumor DNA. ${ }^{4}$ This important suggestion was shown to be correct in 1994 when two groups reported the presence of tumor-associated oncogene mutations in the plasma of patients with myelodysplastic syndrome, acute myelogenous leukemia, ${ }^{5}$ and pancreatic cancer. ${ }^{6}$ These latter reports are powerful evidence that tumor cells can indeed release their DNA into the circulation. Apart from the inherent biological interest of this phenomenon, this observation also opens up exciting possibilities for tumor detection and monitoring.

Significant progress in plasma/serum DNA research had to wait, until 1996, when two groups simultaneously reported the presence of tumor-associated microsatellite alterations in the plasma and serum of cancer patients. ${ }^{7,8}$ Thus, microsatellite alterations, such as loss of heterozygosity ( $\mathrm{LOH})$ and microsatellite shifts, can be found in the plasma and serum and can be shown to match those occurring in the primary tumors. The observation of $\mathrm{LOH}$ in the plasma and serum is particularly impressive as it suggests that, in these cases, most of the plasma/serum DNA is tumor-derived.

\section{TUMOR DNA IN PLASMA AND SERUM}

To date, many tumor-derived genetic alterations have been detected in the plasma and serum. Examples include oncogene mutations, ${ }^{9,10}$ oncogene amplifications, ${ }^{11}$ and tumor-related viral DNA. ${ }^{12-14}$ The development of viral DNA as a tumor marker in plasma/serum has been especially rapid due to the ability to accurately quantify viral DNA in the circulation. Examples include the detection of Epstein-Barr virus (EBV) DNA in the plasma/serum of patients with nasopharyngeal carcinoma, ${ }^{12,15-17}$ lymphoma, ${ }^{18,19}$ and gastric cancer, ${ }^{20}$ and the detection of human papillomavirus (HPV) DNA in the plasma/serum of patients with head and neck cancer ${ }^{14}$ and cervical cancer. ${ }^{21}$ 
As epigenetic changes have now been recognized to be an important molecular change in a wide variety of malignancies, ${ }^{22}$ tumor-associated epigenetic alterations, such as aberrant methylation, have also been observed in the plasma and serum. ${ }^{23-25}$ The availability of quantitative methods for methylation analysis ${ }^{26}$ has made possible the correlation of the concentration of circulating tumor DNA and clinical outcome. $^{27}$

\section{FETAL DNA IN MATERNAL PLASMA/SERUM}

The discovery of circulating DNA of tumoral origin in the plasma also inspired investigators, who were originally interested in other fields, to start looking for other forms of nonhost DNA in the plasma/serum. One such group was initially interested in searching for fetal nucleated cells that have entered into the maternal circulation during pregnancy. ${ }^{28,29}$ By the use of $\mathrm{Y}$ chromosomal DNA sequences that are present in a male fetus as a marker, Lo et al. demonstrated in 1997 the presence of fetal DNA in the plasma/serum of pregnant women. ${ }^{30}$ It is particularly interesting that quantitative analysis has shown that the relative concentration of fetal DNA in maternal plasma is much higher than the corresponding relative concentration of fetal DNA in the cellular fraction. ${ }^{31}$ This finding suggests that maternal plasma fetal DNA would be a very valuable material for noninvasive prenatal diagnosis and monitoring. This possibility has now been realized in a number of fetal and pregnancy-associated disorders. ${ }^{32}$

\section{OTHER FORMS OF “PLASMA DNA CHIMERISM"}

The discovery of circulating tumor-derived, and fetal-derived, DNA in the circulation of cancer patients and pregnant women, respectively, has opened up the concept of "plasma DNA chimerism" in which DNA from cells with detectable genetic differences can be found in the circulation of an individual. This concept is analogous to the well-established concept of cellular chimerism, which has been found in patients following bone marrow or solid organ transplantation ${ }^{33}$ and in a proportion of women following delivery of their children. ${ }^{34}$

The development of the concept of "plasma DNA chimerism" has further prompted investigators to look for other instances of this phenomenon. This line of work has led to the discovery of plasma DNA of donor origin in subjects who have undergone liver or kidney transplantation. ${ }^{35}$ As plasma DNA has been associated with cell death, the measurement of the levels of donor-derived DNA in transplant recipients might allow one to detect graft rejection noninvasively.

DNA from infectious agents represents another possible target for plasma DNA research. The presence of viral nucleic acid in the plasma is, of course, well established, but here we are more interested in nucleic acids from infectious agents that have not previously been detected in the plasma. One example is DNA from Plasmodium, which has recently been detected in the plasma of patients suffering from malaria. ${ }^{36}$ The possible advantages of such plasma-based detection of these infectious agents remain to be established. 


\section{OTHER DIAGNOSTIC DEVELOPMENTS}

The possible relationship between plasma DNA and cell death has been mentioned before. This association has prompted investigators to study the potential diagnostic applications of plasma DNA analysis in conditions associated with cell death. One example is the detection of high concentrations of plasma DNA in patients following trauma. ${ }^{37}$ Excitingly, the levels of plasma DNA in these patients have been shown to be of prognostic significance and may find a role in the routine investigation of trauma patients.

\section{BIOLOGY OF CIRCULATING DNA}

Currently, most research in plasma DNA has focused on the diagnostic application of this phenomenon. However, it is important to realize that there are still many unanswered biological questions in this field. One question concerns the production of plasma DNA. ${ }^{38}$ While cell death has been postulated as one mechanism, ${ }^{39}$ other possibilities have also been suggested, including active secretion. ${ }^{40}$

Apart from production, the clearance of circulating DNA is also poorly understood. Many reports have suggested that circulating DNA is cleared very rapidly from plasma. ${ }^{41,42}$ Potential mechanisms for circulating DNA clearance include plasma nucleases and hepatic and renal clearance. The case for renal clearance is particularly interesting as recent data have shown the presence of tumor- and fetalderived DNA in the urine of cancer patients and pregnant women, respectively. ${ }^{43}$ The situation for cancer patients is especially interesting as it can also be seen in patients with cancers of nonurological origin. These results suggest that plasma DNA can pass through the glomerular barrier and be excreted in the urine. ${ }^{44}$

Another unanswered question concerns the possible functionality of circulating DNA. Intriguing data have reported that, in certain scenarios, circulating DNA may be taken up by cells and result in detectable gene expression. ${ }^{45,46}$

\section{CIRCULATING RNA}

The finding of DNA in the plasma has prompted investigators to look for other types of nucleic acids in the circulation. Such work has led to the simultaneous report by two groups of the existence of tumor-derived RNA in the plasma of cancer patients. ${ }^{47,48}$ These data have now been confirmed by other workers. ${ }^{49}$ Apart from cancer, fetal RNA has also been detected in the plasma of pregnant women. ${ }^{50}$ The theoretical advantage of plasma RNA analysis over plasma DNA detection is that the RNA approach would provide valuable information on the gene expression patterns of the tumor or the fetus. If the technology can be performed robustly and on multiple genes simultaneously, this would allow the performance of noninvasive gene expression profiling by using peripheral blood of cancer patients or pregnant women. 


\section{CONCLUSIONS}

The recent interest in circulating nucleic acids has produced many exciting developments. With further research, this field has the potential to revolutionize medical diagnostics, with new possibilities for the investigation of cancer patients, for performing prenatal diagnosis, and for monitoring transplant and trauma patients. On the biological side, this field has also provided us with new insights into many fundamental biological processes, such as the release and clearance of DNA.

\section{ACKNOWLEDGMENTS}

The author is supported by the Kadoorie Charitable Foundations (under the auspices of the Hong Kong Cancer Genetics Research Group), the Hong Kong Research Grants Council, and the Innovation and Technology Fund (No. AF/90/99).

\section{REFERENCES}

1. Mandel, P. \& P. Métais. 1948. Les acides nucléiques du plasma sanguin chez l'homme. C. R. Acad. Sci. Paris 142: 241-243.

2. TAN, E.M. et al. 1966. Deoxyribonucleic acid (DNA) and antibodies to DNA in the serum of patients with systemic lupus erythematosus. J. Clin. Invest. 45: 1732-1740.

3. LEON, S.A. et al. 1977. Free DNA in the serum of cancer patients and the effect of therapy. Cancer Res. 37: 646-650.

4. Stroun, M. et al. 1989. Neoplastic characteristics of the DNA found in the plasma of cancer patients. Oncology 46: 318-322.

5. VAsioukhin, V. et al. 1994. Point mutations of the N-ras gene in the blood plasma DNA of patients with myelodysplastic syndrome or acute myelogenous leukaemia. Br. J. Haematol. 86: 774-779.

6. SorEnson, G.D. et al. 1994. Soluble normal and mutated DNA sequences from singlecopy genes in human blood. Cancer Epidemiol. Biomarkers Prev. 3: 67-71.

7. CHEN, X.Q. et al. 1996. Microsatellite alterations in plasma DNA of small cell lung cancer patients. Nat. Med. 2: 1033-1035.

8. NAwROZ, H. et al. 1996. Microsatellite alterations in serum DNA of head and neck cancer patients. Nat. Med. 2: 1035-1037.

9. ANKER, P. et al. 1997. K-ras mutations are found in DNA extracted from the plasma of patients with colorectal cancer. Gastroenterology 112: 1114-1120.

10. HiBI, K. et al. 1998. Molecular detection of genetic alterations in the serum of colorectal cancer patients. Cancer Res. 58: 1405-1407.

11. Chiang, P.W. et al. 1999. Detection of erbB-2 amplifications in tumors and sera from esophageal carcinoma patients. Clin. Cancer Res. 5: 1381-1386.

12. Lo, Y.M.D. et al. 1999. Quantitative analysis of cell-free Epstein-Barr virus DNA in plasma of patients with nasopharyngeal carcinoma. Cancer Res. 59: 1188-1191.

13. Mutirangura, A. et al. 1998. Epstein-Barr viral DNA in serum of patients with nasopharyngeal carcinoma. Clin. Cancer Res. 4: 665-669.

14. CAPONE, R.B. et al. 2000. Detection and quantitation of human papillomavirus (HPV) DNA in the sera of patients with HPV-associated head and neck squamous cell carcinoma. Clin. Cancer Res. 6: 4171-4175.

15. Lo, Y.M.D. et al. 1999. Quantitative and temporal correlation between circulating cellfree Epstein-Barr virus DNA and tumor recurrence in nasopharyngeal carcinoma. Cancer Res. 59: 5452-5455.

16. Lo, Y.M.D. et al. 2000. Kinetics of plasma Epstein-Barr virus DNA during radiation therapy for nasopharyngeal carcinoma. Cancer Res. 60: 2351-2355. 
17. Lo, Y.M.D. et al. 2000. Molecular prognostication of nasopharyngeal carcinoma by quantitative analysis of circulating Epstein-Barr virus DNA. Cancer Res. 60: 68786881.

18. LEI, K.I. et al. 2000. Quantitative analysis of circulating cell-free Epstein-Barr virus (EBV) DNA levels in patients with EBV-associated lymphoid malignancies. Br. J. Haematol. 111: 239-246.

19. Gallagher, A. et al. 1999. Detection of Epstein-Barr virus (EBV) genomes in the serum of patients with EBV-associated Hodgkin's disease. Int. J. Cancer 84: 442-448.

20. Lo, Y.M.D. et al. 2001. Circulating Epstein-Barr virus DNA in the serum of patients with gastric carcinoma. Clin. Cancer Res. 7: 1856-1859.

21. Pornthanakasem, W. et al. 2001. Human papillomavirus DNA in plasma of patients with cervical cancer. BMC Cancer 1: 2 .

22. BAYLIN, S.B. et al. 2001. Aberrant patterns of DNA methylation, chromatin formation, and gene expression in cancer. Hum. Mol. Genet. 10: 687-692.

23. Wong, I.H. et al. 1999. Detection of aberrant p16 methylation in the plasma and serum of liver cancer patients. Cancer Res. 59: 71-73.

24. Wong, I.H. et al. 2000. Relationship of p16 methylation status and serum $\alpha$-fetoprotein concentration in hepatocellular carcinoma patients. Clin. Chem. 46: 1420-1422.

25. Wong, I.H. et al. 2000. Frequent p15 promoter methylation in tumor and peripheral blood from hepatocellular carcinoma patients. Clin. Cancer Res. 6: 3516-3521.

26. Lo, Y.M.D. et al. 1999. Quantitative analysis of aberrant p16 methylation using realtime quantitative methylation-specific polymerase chain reaction. Cancer Res. 59: 3899-3903.

27. KAWAKAMI, K. et al. 2000. Hypermethylated APC DNA in plasma and prognosis of patients with esophageal adenocarcinoma. J. Natl. Cancer Inst. 92: 1805-1811.

28. Lo, Y.M.D. et al. 1989. Prenatal sex determination by DNA amplification from maternal peripheral blood. Lancet 2: 1363-1365.

29. Lo, Y.M.D. et al. 1996. Two-way cell traffic between mother and fetus: biologic and clinical implications. Blood 88: 4390-4395.

30. Lo, Y.M.D. et al. 1997. Presence of fetal DNA in maternal plasma and serum. Lancet 350: $485-487$.

31. Lo, Y.M.D. et al. 1998. Quantitative analysis of fetal DNA in maternal plasma and serum: implications for noninvasive prenatal diagnosis. Am. J. Hum. Genet. 62: 768775 .

32. Lo, Y.M.D. 2000. Fetal DNA in maternal plasma: biology and diagnostic applications. Clin. Chem. 46: 1903-1906.

33. StARZL, T.E. et al. 1997. Chimerism after organ transplantation. Curr. Opin. Nephrol. Hypertens. 6: 292-298.

34. BIANCHI, D.W. et al. 1996. Male fetal progenitor cells persist in maternal blood for as long as 27 years postpartum. Proc. Natl. Acad. Sci. U.S.A. 93: 705-708.

35. Lo, Y.M.D. et al. 1998. Presence of donor-specific DNA in plasma of kidney and liver transplant recipients. Lancet 351: 1329-1330.

36. GaL, S. et al. 2001. Detection of Plasmodium falciparum DNA in plasma. Clin. Chem. 47: 370 .

37. Lo, Y.M.D. et al. 2000. Plasma DNA as a prognostic marker in trauma patients. Clin. Chem. 46: 319-323.

38. Stroun, M. et al. 2000. The origin and mechanism of circulating DNA. Ann. N.Y. Acad. Sci. 906: 161-168.

39. FouRNIE, G.J. et al. 1995. Plasma DNA as a marker of cancerous cell death: investigations in patients suffering from lung cancer and in nude mice bearing human tumors. Cancer Lett. 91: 221-227.

40. Rogers, J.C. et al. 1972. Excretion of deoxyribonucleic acid by lymphocytes stimulated with phytohemagglutinin or antigen. Proc. Natl. Acad. Sci. U.S.A. 69: 1685-1689.

41. Tsumita, T. \& M. Iwanaga. 1963. Fate of injected deoxyribonucleic acid in mice. Nature 198: 1088-1089.

42. Lo, Y.M.D. et al. 1999. Rapid clearance of fetal DNA from maternal plasma. Am. J. Hum. Genet. 64: 218-224. 
43. BotezAtU, I. et al. 2000. Genetic analysis of DNA excreted in urine: a new approach for detecting specific genomic DNA sequences from cells dying in an organism. Clin. Chem. 46: $1078-1084$

44. Lo, Y.M.D. 2000. Molecular testing of urine: catching DNA on the way out. Clin. Chem. 46: 1039-1040.

45. Garcia-Olmo, D. et al. 1999. Tumor DNA circulating in the plasma might play a role in metastasis: the hypothesis of the genometastasis. Histol. Histopathol. 14: 1159-1164.

46. Holmgren, L. et al. 1999. Horizontal transfer of DNA by the uptake of apoptotic bodies. Blood 93: 3956-3963.

47. Lo, K.W. et al. 1999. Analysis of cell-free Epstein-Barr virus associated RNA in the plasma of patients with nasopharyngeal carcinoma. Clin. Chem. 45: 1292-1294.

48. KopRESKI, M. et al. 1999. Detection of tumor messenger RNA in the serum of patients with malignant melanoma. Clin. Cancer Res. 5: 1961-1965.

49. CHEN, X.Q. et al. 2000. Telomerase RNA as a detection marker in the serum of breast cancer patients. Clin. Cancer Res. 6: 3823-3826.

50. Poon, L.L. et al. 2000. Presence of fetal RNA in maternal plasma. Clin. Chem. 46: $1832-1834$ 